\title{
Effects of Geometric Azimuthal Asymmetries of the PPM Stack on Electron Beam Characteristics*
}

\author{
Carol L. Kory \\ ANALEX Corporation/NASA Glenn Research Center \\ 21000 Brookpark Road, MS 54-5 \\ Cleveland, Ohio 44135 \\ (216) 433-3512 Tel \\ (216) 433-8705 Fax \\ Carol.L.Kory@grc.nasa.gov
}

The effects of geometric azimuthally asymmetric properties of a periodic permanent magnet (PPM) focusing stack on electron beam characteristics obtained using a fully threedimensional (3D) particle-in-cell (PIC) code will be presented. The simulation model, using MAFIA (Solution of MAxwell's equations by the Finite-Integration-Algorithm), incorporates 3D behavior of the beam immersed in static fields calculated directly from the exact geometry and material properties of the 3D magnetic focusing structure [1]. The Hughes $8916 \mathrm{H}, 18-40 \mathrm{GHz}$ helical TWT for the millimeter-wave power module (MMPM) was used as a prototype [2].

Firstly, the effects of C-magnets used at the input and output of the TWT to allow for coupling of the RF signal into and out of the tube are considered. The $8916 \mathrm{H}$ input and output C-magnets differ because coaxial couplers are used at the input and waveguide couplers are used at the output. The repositioning of the beam from its central axis due to the inclusion of the output C-magnet was found to be most significant. The modeled output $\mathrm{C}$-magnet and its orientation in the Cartesian coordinate system is shown in Figure 1, and a two-dimensional beam profile including the output C-magnet is shown in Figure 3. Table I presents the shift of the beam center off the central axis relative to the average radius of the beam at the longitudinal points $\mathrm{A}, \mathrm{B}$ and $\mathrm{C}$ designated on Figure 3.

Secondly, the addition of shunts, or rectangular iron pieces applied manually by a skilled technician in order to improve beam transmission, is considered. The shunts are applied to the top of the tube; thus, azimuthal symmetry of the focusing stack is interrupted. Although shunts are typically added during RF focusing, they are also typically added at the input section of the tube where RF forces are minimal, making an electron optics analysis meaningful. Because several shunts are usually applied to one pole piece, the simulations have been simplified by modeling a half washer with the same radius and longitudinal length as a shunt over the entire $x$, positive-y half of the transverse plane. A modeled pole piece and shunt as described are shown in Figure 2.

Lastly, in order to study the effects of magnet misalignments, a magnet in the PPM stack was arbitrarily chosen and adjusted so that its central axis was shifted both 0.7 percent and 1.0 percent of the magnet outer diameter in the positive-y direction. In practice, positioning the magnets so that their central axis is accurately aligned with the central axis of the tube is challenging. Thus, it is a strong possibility that one or more magnets will be misaligned relative to the tube central axis.

The effects of the described geometrically asymmetric conditions on the electron beam will be presented quantitatively in terms of beam position, percent ripple and percent transmission.

1. C. L. Kory and A. Haq Qureshi, Three-Dimensional Simulation of Periodic Permanent Magnetic Focusing of Electron Beams, NASA Contractor Report in preparation, 2000.

2. Personal communication with Will Menninger of Hughes Aircraft Co., Electron Dynamics Division.

* This work is in support of the NASA Cross Enterprise Technology Development Program in Code SM. 


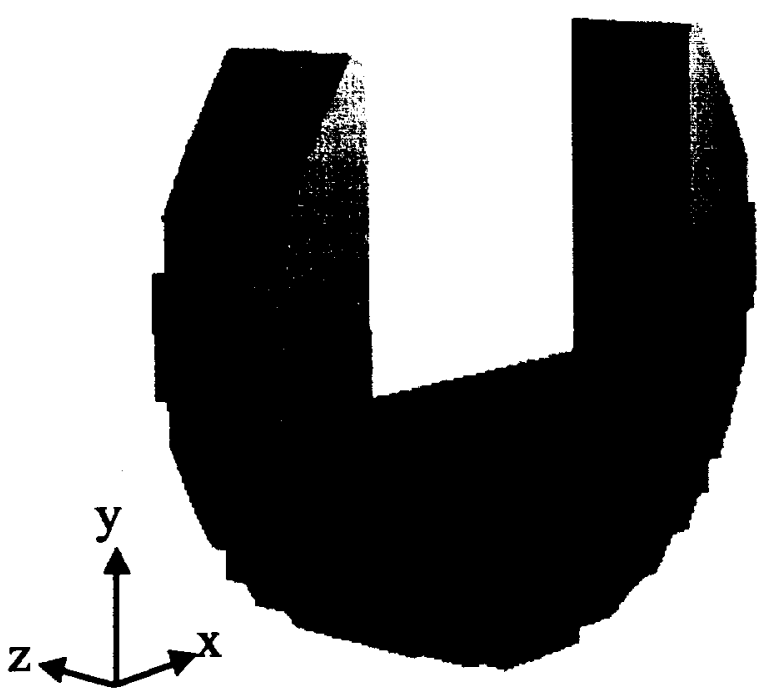

Figure 1 Simulated output C-magnet

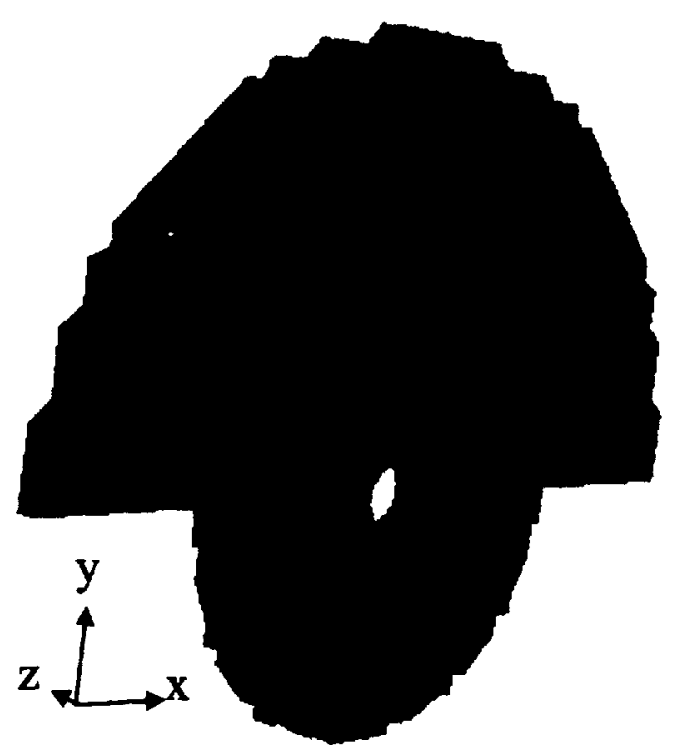

Figure 2 Simulated pole piece with shunt approximated by half-washer

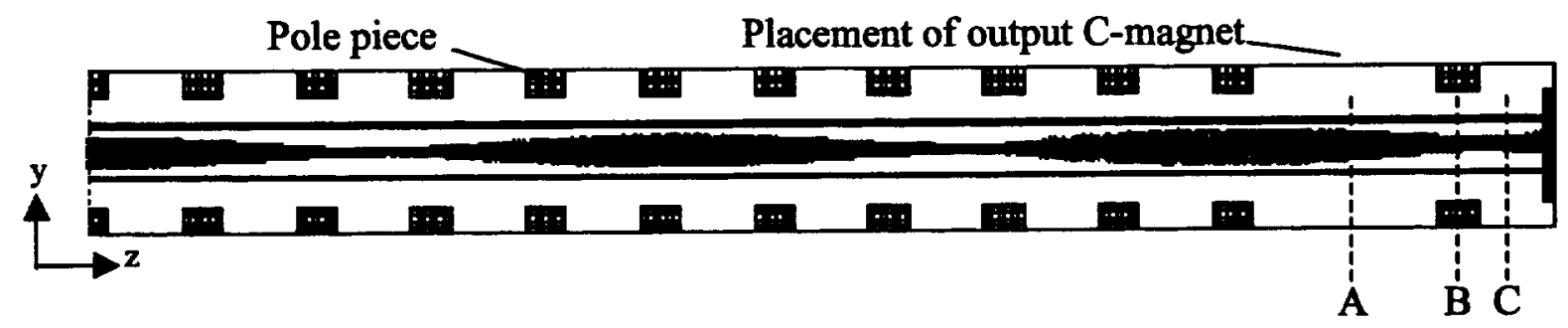

Figure 3 Simulated two-dimensional beam profile over six magnetic periods for $81 \mathrm{~mA}$ beam using twice the Brillouin field including output $\mathrm{C}$-magnet

Table I Simulated beam shift off central axis at longitudinal points A, B and C of Figure 3 due to inclusion of output $\mathrm{C}$-magnet.

\begin{tabular}{|c|c|c|}
\hline $\begin{array}{c}\text { Longitudinal } \\
\text { Position }\end{array}$ & \multicolumn{2}{|c|}{$\begin{array}{c}\text { Shift off central axis } \\
\text { (percent of average beam radius) }\end{array}$} \\
\cline { 2 - 3 } & x-direction & y-direction \\
\hline A & 8 & 6 \\
\hline B & 2 & 24 \\
\hline C & -8 & 20 \\
\hline
\end{tabular}

